\title{
Corticocortical Interactions between and within Three Cortical Auditory Areas Specialized for Time-Domain Signal Processing
}

\author{
Jie Tang and Nobuo Suga \\ Department of Biology, Washington University, St. Louis, Missouri 63130
}

In auditory cortex of the mustached bat, the $\mathrm{FF}$ ( $\mathrm{F}$ means frequency modulation), dorsal fringe (DF), and ventral fringe (VF) areas consist of "combination-sensitive" neurons tuned to the pair of an emitted biosonar pulse and its echo with a specific delay (best delay: BD). The DF and VF areas are hierarchically at a higher level than the FF area. Focal electric stimulation of the FF area evokes "centrifugal" BD shifts of DF neurons, i.e., shifts away from the BD of the stimulated FF neurons, whereas stimulation of the DF neurons evokes "centripetal" BD shifts of FF neurons, i.e., shifts toward the BD of the stimulated DF neurons. In our current studies, we found that the feedforward projection from FF neurons evokes centrifugal BD shifts of VF neurons, that the feedback projection from VF neurons evokes centripetal BD shifts of FF neurons, that the contralateral projection from DF neurons evokes centripetal BD shifts of DF neurons, and that the centripetal BD shifts evoked by the DF and VF neurons are 2.5 times larger than the centrifugal BD shifts evoked by the FF neurons. The centrifugal BD shifts shape the selective neural representation of a specific target distance, whereas the centripetal BD shifts expand the representation of the selected specific target distance to focus on the processing of the target information at a specific distance. The centrifugal and centripetal BD shifts evoked by the feedforward and feedback projections promote finer analysis of a target at shorter distances.

\section{Introduction}

The auditory cortex (AC) consists of multiple, areas as does the visual cortex. However, the functional organization of the cortical auditory areas beyond tonotopy has been poorly explored, except for the AC of the mustached bat, Pteronotus parnellii (Fig. $1 A$ ). It has been known that tonotopically organized cortical auditory areas are commonly interconnected (for review, see Rouiller, 1997). In the mustached bat, the corticocortical interaction within the primary auditory cortex has been studied (for review, see Suga and Ma, 2003). However, the corticocortical interaction between the cortical auditory areas has been only recently studied by Tang and Suga (2008).

In the mustached bat, the target distance information carried by echo delays is represented in three cortical areas: the FF ( F means frequency modulation), dorsal fringe (DF), and ventral fringe (VF) areas. [The FF area has been called the FM-FM area because it consists of FM-FM combination-sensitive neurons. Both the DF and VF areas, subsequently found, also consist of FM-FM neurons. Therefore, the FM-FM area has recently been changed to the FF area (Tang and Suga, 2008).] In these three areas, neurons are tuned to specific echo delays and form an echo-delay axis for the systematic neural representation of target

Received Jan. 23, 2009; revised March 31, 2009; accepted May 6, 2009.

This work was supported by a research grant from the National Institute on Deafness and Other Communication Disorders (DC-000175). We thank S. E. Miller for editing our current manuscript.

Correspondence should be addressed to Nobuo Suga, Department of Biology, Washington University, St. Louis, M0 63130. E-mail: suga@biology.wustl.edu.

DOI:10.1523/JNEUROSCI.0373-09.2009

Copyright $\odot 2009$ Society for Neuroscience $\quad$ 0270-6474/09/297230-08\$15.00/0 distances. That is, the best delays (BDs) for their excitation systematically vary along the cortical surface. The size and the echodelay axis of the DF and VF areas are smaller and shorter than those of the FF area (Fig. 1 B) (for review, see Suga, 1990). The DF and VF areas are at higher levels than the FF area in terms of information flow. These three areas are heavily connected with each other (Fitzpatrick et al., 1998).

Focal electric stimulation of the FF area augments surrounding FF neurons which match the stimulated neurons in BD and sharpen their delay tuning, whereas it inhibits other surrounding neurons that are unmatched in BD and shifts their delay tuning, i.e., shifts their BDs away from the BD of the stimulated FF neurons (Xiao and Suga, 2004). Such a shift in tuning is called a "centrifugal" shift (Suga et al., 2000). On the other hand, focal electric stimulation of the DF area shifts the BDs of surrounding neurons toward that of the stimulated DF neurons (Tang and Suga, 2008). Such a shift in tuning is called a "centripetal" shift (Suga et al., 2002). However, the corticocortical interactions between different cortical auditory areas have thus far been studied only between DF and FF areas. Electric stimulation of FF neurons evokes centrifugal BD shifts of DF neurons through the feedforward projection, whereas electric stimulation of DF neurons evokes centripetal BD shifts of the FF neurons through the feedback projection.

In our current studies, we found that electric stimulation of FF neurons evoked centrifugal BD shifts of VF neurons, whereas that of VF neurons evoked centripetal BD shifts of FF neurons, that electric stimulation of DF neurons evoked centripetal BD shifts of 

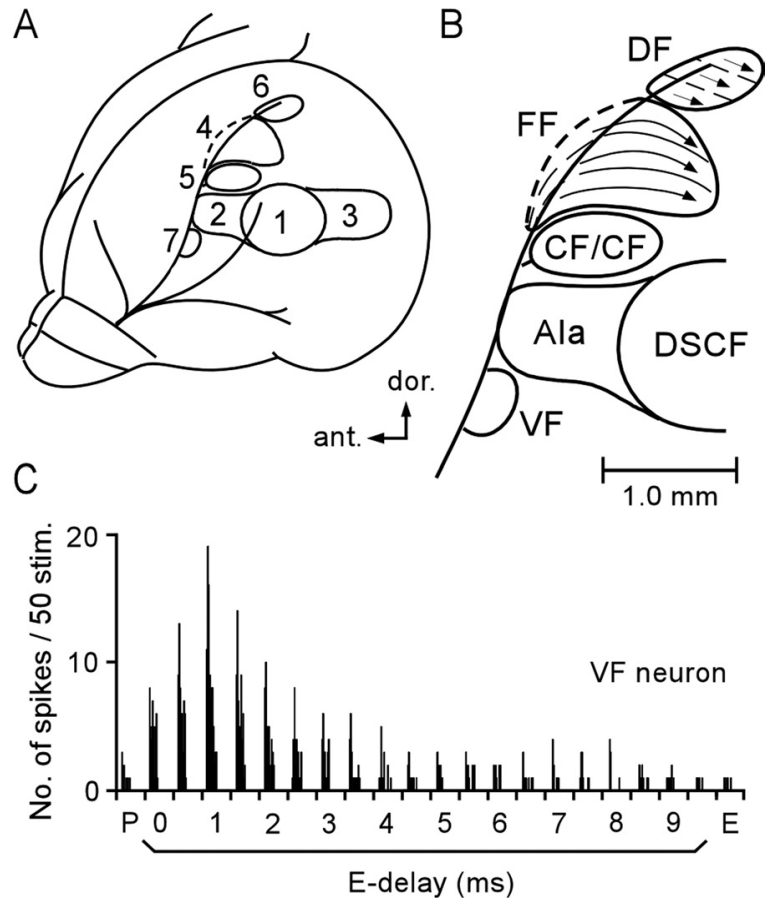

Figure 1. The auditory cortex, the delay-tuned areas, and the responses of a delay-tuned neuron. $A$, Dorsolateral view of the left cerebral cortex of the mustached bat. "1-7" are respectively the DSCF, Ala (anterior division of Al), Alp (posterior division of Al), FF, CF/CF, DF, and VF areas. The FF area was previously called the FM-FM area. $\boldsymbol{B}$, The FF, DF, and VF areas each consist of three types of combination-sensitive neurons called $\mathrm{FM}_{1}-\mathrm{FM}_{2}, \mathrm{FM}_{1}-\mathrm{FM}_{3}$, and $\mathrm{FM}_{1}-$ $\mathrm{FM}_{4}$ delay-tuned neurons. The subscripts 1-4 indicate the first through fourth harmonics of FM components of biosonar pulses and echoes. $C$, The array of PST histograms displaying the responses of a VF neuron to pulse $F M_{1}$ alone $(P)$, echo $F M_{2}$ alone $(E)$, and $P-E$ pairs with different echo (E) delays ranging from 0.0 to $9.5 \mathrm{~ms}$. The best delay for the facilitatory response of the neuron was $1.0 \mathrm{~ms}$

the contralateral DF neurons, and that feedback modulation was 2.5 times stronger than feedforward modulation.

\section{Materials and Methods}

General. Surgery, acoustic and electric stimulation, recording of action potentials, and data acquisition and processing were the same as those previously described (Xiao and Suga, 2004; Tang and Suga, 2008). The only difference was the locations of the cortical sites of the electric stimulation and recording action potentials. Therefore, only the essential portions of the methods are described below. The Animal Studies Committee of Washington University in St. Louis approved the protocol for the present research.

Seven adult mustached bats (Pteronotus parnellii rubiginosus) from Trinidad were used. Under neuroleptanalgesia (Innovar $4.08 \mathrm{mg} / \mathrm{kg}$ of body weight), a 1.5-cm-long metal post was glued on the dorsal surface of the bat's skull. A local anesthetic (lidocaine $\mathrm{HCl}$ ) and antibiotic ointment (Furacin) were applied to the surgical wound. Three to four days after surgery, the awake animal was placed in a polyethylene-foam body mold, which was hung with an elastic band at the center of a $31^{\circ} \mathrm{C}$ soundproof room. The metal post glued on the skull was attached to a metal rod with set screws to immobilize the animal's head, which was adjusted directly toward the loudspeakers located $74 \mathrm{~cm}$ away. A few holes $(50-100 \mu \mathrm{m}$ in diameter) were made in the skull covering the FF, VF, and/or DF areas of the right and left auditory cortices. A pair of tungsten-wire electrodes ( $\sim 7 \mu \mathrm{m}$ in tip diameter, $\sim 35 \mu \mathrm{m}$ apart, one proximal to the other) was orthogonally inserted through one of the holes, 500-700 $\mu$ m deep into one of these three areas. The responses (action potentials) of delay-tuned neurons to pairs of FM sounds were recorded and the BD for excitation of the neurons was measured. Then, this electrode pair was used to electrically stimulate the neurons. A single glass micropipette electrode $(\sim 1$ $\mu \mathrm{m}$ in tip diameter) was inserted into one of these three areas to record the responses of other delay-tuned neurons to the pairs of FM sounds and to examine the effect of the electrical stimulation on these responses.

Acoustic stimulation. The mustached bat emits orientation sounds (biosonar pulses or, simply, pulses). Each pulse consists of constantfrequency (CF) and frequency-modulated (FM) components, each of which contains four harmonics. Therefore, each pulse contains eight components $\left(\mathrm{CF}_{1-4}, \mathrm{FM}_{1-4}\right)$. Neurons in FF, DF, and VF areas are tuned to a combination of the $\mathrm{FM}_{1}$ of the pulse stimulus and $\mathrm{FM}_{n}(n=2,3$, or 4) of an echo stimulus with a specific time delay from the pulse stimulus. Different types of delay-tuned neurons $\left(\mathrm{FM}_{1}-\mathrm{FM}_{2}, \mathrm{FM}_{1}-\mathrm{FM}_{3}\right.$, and $\mathrm{FM}_{1}-$ $\mathrm{FM}_{4}$ ) are separately clustered in these three areas (Fig. 1B) (Suga and O’Neill, 1979; O'Neill and Suga, 1982; Edamatsu et al., 1989). Therefore, acoustic stimuli delivered to the animal were $\mathrm{FM}_{1}-\mathrm{FM}_{n}$ pairs. Each FM sound was $3 \mathrm{~ms}$, including a $0.5 \mathrm{~ms}$ rise-delay time.

To generate an FM sound, a voltage-controlled oscillator (Wavetek 134) was frequency-modulated with a linear voltage-ramp generator to mimic the FM components in the species-specific biosonar pulse. The frequency-modulation and the amplitude of each FM sound and the time interval (echo delay) between paired FM sounds were first manually varied to identify the best combination of two FM sounds $\left(\mathrm{FM}_{1}-\mathrm{FM}_{n}\right)$ and the $\mathrm{BD}$ to excite a given neuron. Then, the $\mathrm{FM}_{1}$ sound (pulse stimulus) was fixed at the frequency sweep and amplitude to evoke the largest facilitation. The $\mathrm{FM}_{n}$ sound (echo stimulus) was fixed at the frequency sweep to evoke the largest facilitation and at $10 \mathrm{~dB}$ above the minimum threshold, i.e., the threshold of the response at the best $\mathrm{FM}_{n}$. Then, the $\mathrm{FM}_{n}$ delay from $\mathrm{FM}_{1}$ was varied with a computer and other hardware (Tucker-Davis Technologies) to obtain delay-response curves. The computer-controlled delay scan consisted of twenty-two $150 \mathrm{~ms}$ time blocks: pulse only, echo only, and 20 pulse-echo pairs in which the echo delay randomly varied from $0 \mathrm{~ms}$ to $9.5 \mathrm{~ms}$ in $0.5 \mathrm{~ms}$ steps. An identical delay scan was delivered 50 times to obtain an array of poststimulus time (PST) or PST cumulative histograms displaying the responses of a single neuron.

Electric stimulation of cortical neurons. Electric stimulation was a monophasic electric pulse ( $0.2 \mathrm{~ms}$ long, $100 \mathrm{nA})$ delivered at a rate of 5/s for 7 min with a constant current stimulator (WPI, modified model A360). Such electric pulses delivered at a low rate evoke changes in the cortical, thalamic, and collicular FM-FM (Yan and Suga, 1996, 1998) and Doppler-shifted constant-frequency (DSCF) (Zhang et al., 1997; Xiao and Suga, 2002) neurons, but do not evoke any noticeable changes in the cochlear microphonic responses (Xiao and Suga, 2002). These electric pulses were estimated to activate neurons within a $60 \mu \mathrm{m}$ radius in the plane orthogonal to the cortical columns (Yan and Suga, 1996).

The FF area is $1.4 \mathrm{~mm} \times 0.9 \mathrm{~mm}\left(\sim 0.9 \mathrm{~mm}^{2}\right)$ and consists of three elongated subdivisions: $\mathrm{FM}_{1}-\mathrm{FM}_{2}, \mathrm{FM}_{1}-\mathrm{FM}_{4}$, and $\mathrm{FM}_{1}-\mathrm{FM}_{3}$, which are arranged ventral to dorsal in this order. Each of the three subdivisions of the $\mathrm{FF}$ area has a delay (time) axis, and the iso- $\mathrm{BD}$ lines in one subdivision are in register with those in the other subdivisions (Suga and O'Neill, 1979; Misawa and Suga, 2001). Each subdivision is $\sim 0.3 \mathrm{~mm}$ wide. Therefore, each subdivision could be selectively stimulated with the 0.2 $\mathrm{ms}, 100 \mathrm{nA}$ electric pulses. On the other hand, the VF area is located at the anteroventral edge of the auditory cortex. It is very small $\left(\sim 0.1 \mathrm{~mm}^{2}\right)$, and it is unknown whether it consists of three subdivisions in terms of combination sensitivity. Therefore, there was a possibility that the different types of FM-FM neurons in the VF area were simultaneously activated by the electric stimulation.

Data acquisition. Action potentials of a single cortical delay-tuned neuron tuned to a pair of $\mathrm{FM}_{1}-\mathrm{FM}_{n}$ sounds with a specific $\mathrm{FM}_{n}$ delay from $\mathrm{FM}_{1}$ were selected with a time-amplitude-window discriminator software (Tucker-Davis Technologies). At the beginning of data acquisition, the waveform of an action potential was stored and displayed on the computer monitor screen. This action potential (i.e., template) was compared with other action potentials obtained during data acquisition. The response of the single cortical neuron to a delay scan delivered 50 times was recorded before and after electric stimulation, and was displayed as an array of PST or PST cumulative histograms. The data were stored in the computer hard-drive and were used for off-line analysis. In a $1 \mathrm{~d}$ 
experiment, only one or two neurons were studied for the effect of and recovery from electric stimulation.

Evoked potentials of a small group of delaytuned neurons elicited by pairs of $\mathrm{FM}_{1}-\mathrm{FM}_{n}$ sounds were recorded by Brainware (TDT system 3, gain 5000-15,000, bandpass 100-300 $\mathrm{Hz}$ ). An averaged evoked potential was based on 400 trials. It was displayed on the computer monitor screen and saved for later off-line analysis.

Off-line data processing. The magnitude of auditory responses of a neuron was expressed by the number of spikes per 50 identical stimuli and was plotted as a function of echo delays. The BD shift evoked by the cortical electric stimulation was considered significant if it shifted back (i.e., recovered) to the BD in the control condition and if the auditory responses changed by the electric stimulation recovered by $>85 \%$. The one-way ANOVA was used to examine the difference between the delaytuning curves obtained before and after the electric stimulation. The confidence level was taken as $95 \%(p<0.05)$.

\section{Results}

Since the FF, DF, and VF areas are respectively $\sim 0.9, \sim 0.6$, and $\sim 0.1 \mathrm{~mm}^{2}$, recording of single-unit responses to paired FM sounds was not difficult from the FF and DF areas, but was difficult from the VF area. The number of stimulated and/or recorded neurons was 39, 14, and 39 for the FF, DF, and VF areas, respectively. All these neurons were tuned to a specific echo delay. As an example, Figure $1 C$ shows the responses of a VF neuron tuned to a $1.0 \mathrm{~ms}$ echo delay by an array of PST histograms.

The neuron responded to a pulse stimulus (P) alone and an echo (E) stimulus alone by discharging 0.16 and 0.10 spike per stimulus, respectively. When these stimuli were paired, however, the neuron showed a strong facilitatory response by discharging 1.82 spikes per stimulus at its best delay, $1.0 \mathrm{~ms}$.

\section{Feedforward modulation of VF neurons by FF neurons}

In four animals, the responses of 16 single VF neurons and five evoked potentials at different locations were recorded before and after the electric stimulation of ipsilateral FF neurons. The combination sensitivity of the recorded VF neurons was either $\mathrm{FM}_{1}-$ $\mathrm{FM}_{2}$ (10 neurons and 4 evoked potentials), $\mathrm{FM}_{1}-\mathrm{FM}_{3}$ (2 neurons), or $\mathrm{FM}_{1}-\mathrm{FM}_{4}$ ( 4 neuron and 1 evoked potential). Their BDs ranged from $1.0 \mathrm{~ms}$ to $4.5 \mathrm{~ms}$ (mean $\pm \mathrm{SE}, 2.3 \pm 0.3 \mathrm{~ms}, n=21$ ). The combination sensitivity of the stimulated FF neurons was either $\mathrm{FM}_{1}-\mathrm{FM}_{2}$ (19 neurons) or $\mathrm{FM}_{1}-\mathrm{FM}_{4}$ (2 neurons). Their $\mathrm{BDs}$ ranged from $0.5 \mathrm{~ms}$ to $8.0 \mathrm{~ms}(4.7 \pm 0.5 \mathrm{~ms}, n=21)$. All 16 VF neurons and five evoked potentials studied had a BD that was different from that of the stimulated FF neurons by $>0.5 \mathrm{~ms}$, so that they were BD-unmatched.

Electric stimulation of FF neurons inhibited or facilitated the responses of the BD-unmatched VF neurons and evoked potentials to pulse-echo pairs, depending on the echo delays and the relationship in the BD between the recorded VF neurons or evoked potentials and the stimulated FF neurons. These delaydependent response changes caused the BDs of the VF neurons onset of the FF stimulation.
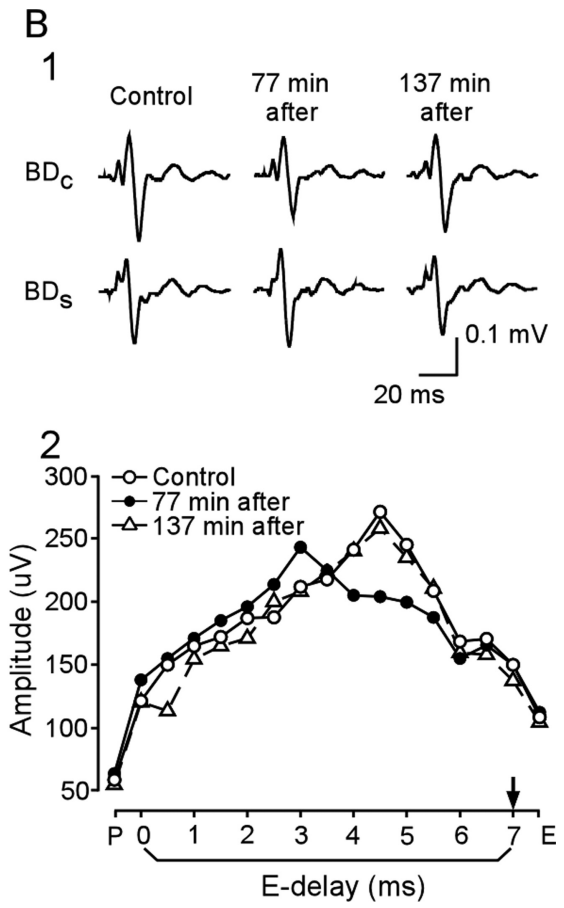

Figure 2. Changes in the responses and delay-response curves of a single VF neuron and a VF evoked potential evoked by (open circles, control), $85 \mathrm{~min}$ after (filled circles), and $137 \mathrm{~min}$ after (open triangles, dashed line) the onset of the FF potential obtained before (open circles, control), $77 \mathrm{~min}$ after (filled circles), and $137 \mathrm{~min}$ after (open triangles, dashed line) the

and evoked potentials to shift away from the BDs of the stimulated FF neurons. That is, the BD shifts were centrifugal.

For example, electric stimulation of FF neurons tuned to a 7.0 ms delay decreased the response of a VF neuron tuned to a $3.0 \mathrm{~ms}$ delay at its BD (Fig. 2A1, upper PST histograms) and increased it at a $1.0 \mathrm{~ms}$ delay (Fig. 2A1, lower PST histograms). Because of these delay-dependent inhibition and facilitation, the $\mathrm{BD}$ of the VF neuron shifted from $3.0 \mathrm{~ms}$ to $1.0 \mathrm{~ms}$ (Fig. 2 A2). These delaydependent changes disappeared $\sim 137$ min after the onset of the electric stimulation (Fig. $2 A 1, A 2$ ). The mean recovery time of the BD shift was $109 \pm 6.3$ min for the 16 VF neurons studied.

Because of the small size of the VF area, recording the action potentials of a single neuron from this area was difficult, but recording the evoked potential tuned to a specific BD was not very difficult. In such a case, we studied the effect of the FF stimulation on the VF evoked potential. Figure 2 B1 shows the evoked potentials at their BDs in the control $\left(\mathrm{BD}_{\mathrm{c}}\right)$ and shifted $\left(\mathrm{BD}_{\mathrm{s}}\right)$ conditions. The evoked potential at the $\mathrm{BD}_{\mathrm{c}}$ decreased after electric stimulation of the FF neurons tuned to a $7.0 \mathrm{~ms}$ delay, and that at the $\mathrm{BD}_{\mathrm{s}}$ increased after the stimulation. The delay-tuning curve of the evoked potential was generally broader than that of a single neuron. Despite that, it was clear that these delaydependent amplitude changes resulted in the centrifugal BD shift from $4.5 \mathrm{~ms}$ to $3.0 \mathrm{~ms}$. This BD shift is clearly shown by the delay-tuning curves of the evoked potential in Figure 2 B2 (filled circles). The delay-dependent changes disappeared $\sim 137$ min after the electric stimulation (Fig. $2 B 1, B 2$ ). The mean recovery 

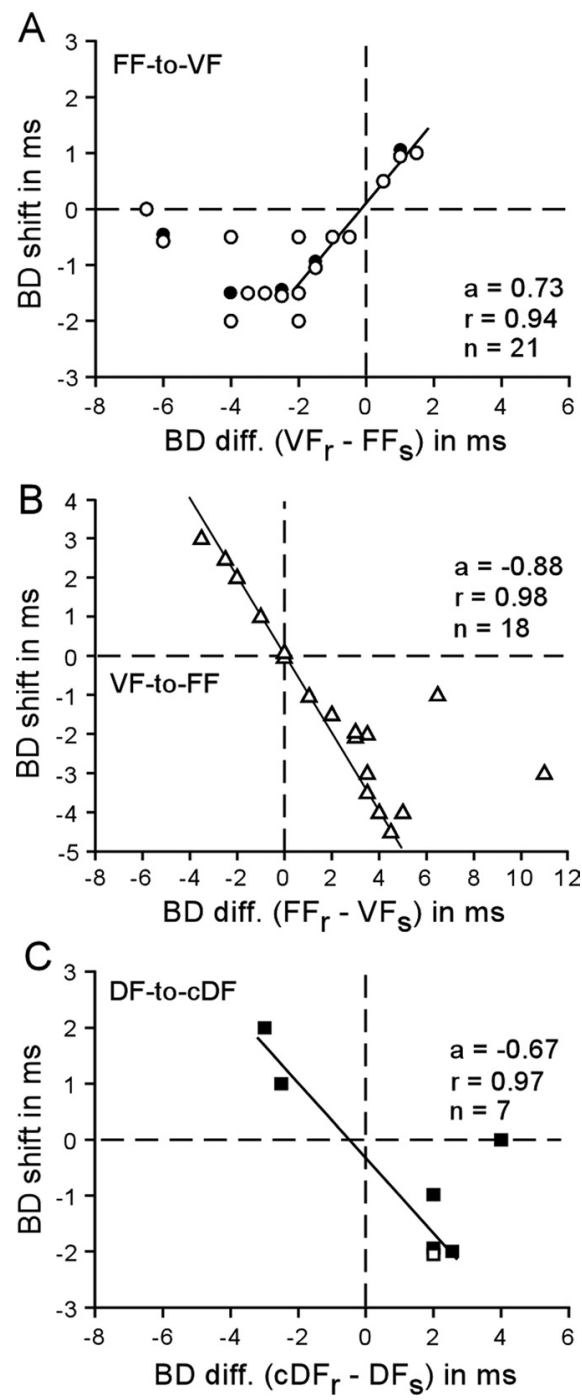

Figure 3. BD shifts as a function of the BD difference between the recorded and stimulated neurons. $A$, The BD shifts of 16 VF neurons (open circles) and five evoked potentials (filled circles) evoked by electric stimulation of $\mathrm{FF}$ neurons. FF-to-VF, A feedforward projection from the FF area to the VF area. $\boldsymbol{B}$, The BD shifts of $18 \mathrm{FF}$ neurons (triangles) evoked by electric stimulation of VF neurons. VF-to-FF, A feedback projection from the VF area to the FF area. $C$, The BD shifts of one contralateral DF neuron (open square) and six contralateral DF evoked potentials (filled squares) evoked by electric stimulation of DF neurons. DF-to-CDF, Projection from the DF area to the contralateral DF area.

time of the BD shift evoked by the FF stimulation was $119.6 \pm$ $11.1 \mathrm{~min}$ for the five VF evoked potentials. This recovery time was statistically not different from that for the 16 single VF neurons $(p=0.16)$. Because the evoked potential originated from the responses of many VF neurons, its centrifugal BD shift indicated that the FF stimulation centrifugally shifted the BDs of many VF neurons.

The BD shifts of the 16 single neurons and five evoked potentials were plotted as a function of the BD differences between the recorded VF and stimulated FF neurons (Fig. $3 A$ ). In Figure $3 A$, the BDs of three single VF neurons and one VF evoked potential were larger than those of the stimulated FF neurons. Their BDs increased after the FF stimulation. On the other hand, the BDs of 12 single VF neurons and four VF evoked potentials were smaller than those of the stimulated FF neurons. Their BDs decreased after the FF stimulation. Therefore, the BD shifts were centrifugal regardless of whether the BDs of the VF neurons were larger or smaller than those of the stimulated FF neurons. The amount of the BD shifts was linearly related to the BD differences within $\pm 2.0 \mathrm{~ms}(\mathrm{a}=0.73 ; r=0.94)$, but it plateaued at -2 to $-4 \mathrm{~ms} \mathrm{BD}$ differences. Then it reduced to zero at a $7.0 \mathrm{~ms}$ BD difference. The largest BD shift observed was $2.0 \mathrm{~ms}$. The feedforward modulation from the FF neurons was always centrifugal to the VF neurons. The asymmetrical distribution of the $\mathrm{BD}$ shifts of the VF neurons along the BD difference axis was due to the short delay axis represented in the VF area (up to $4.5 \mathrm{~ms}$ ) compared with the long delay axis represented in the FF area (up to $18 \mathrm{~ms}$ ). The BD differences longer than $2.0 \mathrm{~ms}$ were not recorded.

Of the 21 pairs of the stimulated FF and recorded VF neurons, FM-FM combination sensitivity was different in seven pairs, but all the VF neurons showed a centrifugal BD shift regardless of combination sensitivity. In two pairs of the seven, the stimulated $\mathrm{FF}$ and recorded VF neurons were $\mathrm{FM}_{1}-\mathrm{FM}_{2}$ and $\mathrm{FM}_{1}-\mathrm{FM}_{3}$, respectively. The $\mathrm{FM}_{1}-\mathrm{FM}_{2}$ and $\mathrm{FM}_{1}-\mathrm{FM}_{3}$ neurons in the FF area were separated from each other by $\mathrm{FM}_{1}-\mathrm{FM}_{4}$ neurons by at least $300 \mu \mathrm{m}$, so the stimulation of the $\mathrm{FM}_{1}-\mathrm{FM}_{2}$ neurons did not stimulate the $\mathrm{FM}_{1}-\mathrm{FM}_{3}$ neurons in the FF area by spreading the stimulus current, but modulated the $\mathrm{FM}_{1}-\mathrm{FM}_{3}$ neurons in the VF area. Therefore, our data indicate that, as the FF-to-DF feedforward modulation (Tang and Suga, 2008), the FF-to-VF feedforward modulation occurred regardless of the differences in the types of FM-FM combination sensitivities between the recorded and stimulated neurons.

Recording of BD-matched VF neurons was difficult, so that they were not included in our current samples. However, it is reasonable to conclude that they do not show BD shifts because the regression line $(r=0.94)$ in Figure $3 A$ crosses the origin of the coordinates as in our previous studies on the interaction between the FF and DF areas (Tang and Suga, 2008).

\section{Feedback modulation of FF neurons by VF neurons}

In five bats, the delay-response curves of $18 \mathrm{FF}$ neurons were studied before and after electric stimulation of ipsilateral VF neurons. The combination sensitivity of the recorded neurons was $\mathrm{FM}_{1}-\mathrm{FM}_{2}$ (16 neurons) or $\mathrm{FM}_{1}-\mathrm{FM}_{4}$ (2 neurons). They were tuned to echo delays ranging from $1.0 \mathrm{~ms}$ to $13 \mathrm{~ms}$ (mean $\pm \mathrm{SE}$, $4.3 \pm 0.65 \mathrm{~ms}$ ). The combination sensitivity of the stimulated VF neurons was $\mathrm{FM}_{1}-\mathrm{FM}_{2}$ (11 neurons), $\mathrm{FM}_{1}-\mathrm{FM}_{3}$ (4 neurons), or $\mathrm{FM}_{1}-\mathrm{FM}_{4}$ (3 neurons). Of the $18 \mathrm{FF}$ neurons, 16 had BDs that were different by $>0.5 \mathrm{~ms}$ from those of the stimulated VF neurons, so that they were $\mathrm{BD}$-unmatched neurons. The remaining two neurons had BDs that were the same as those of the stimulated VF neurons, so that they were BD-matched neurons.

Electric stimulation of the unmatched VF neurons also evoked delay-dependent changes in the responses of the FF neurons. However, the best frequency (BF) shift was centripetal. When the VF neurons tuned to a $2.0 \mathrm{~ms}$ delay were electrically stimulated, for example, the response of the FF neuron tuned to a $3.5 \mathrm{~ms}$ delay decreased at this delay (Fig. $4 \mathrm{Al}, \mathrm{BD}_{\mathrm{c}}$ ), but increased at a 2.0 ms delay (Fig. $4 A 1, \mathrm{BD}_{\mathrm{s}}$ ). Because of this delay-dependent inhibition and facilitation, the BD of the FF neuron shifted from $3.5 \mathrm{~ms}$ to $2.0 \mathrm{~ms}$ (Fig. 4 A2, filled circles). That is, the BD shift was centripetal. These changes disappeared, i.e., the $\mathrm{BD}$ and tuning curve reverted to those in the control condition, $160 \mathrm{~min}$ after the onset of the electric stimulation (Fig. 4A2, dashed line). All BDunmatched FF neurons studied showed the centripetal BD shift for the electric stimulation of the VF neurons.

Of the 18 pairs of the stimulated VF and recorded FF neurons, FM-FM combination sensitivity was different in seven pairs. However, all BD-unmatched FF neurons showed a centripetal BF 
shift regardless of combination sensitivity (see Discussion). The amount of centripetal BD shifts was linearly related to the differences in $\mathrm{BD}$ between the recorded and stimulated neurons (Fig. 3B). The larger the $\mathrm{BD}$ difference, the larger the BD shift. The largest BD shift observed was $4.5 \mathrm{~ms}$, for which the BD difference was $4.5 \mathrm{~ms}$. Such a large BF shift has not yet been observed for the FF stimulation (Xiao and Suga, 2004; Tang et al., 2007; Tang and Suga, 2008). The slope of the BD shiftdifference curve was $-0.88(r=0.98, n=$ 18). Beyond a $5.0 \mathrm{~ms} \mathrm{BD}$ difference, the BD shift became smaller. The mean recovery time of the BD shifts of the FF neurons evoked by the VF stimulation was $113 \pm$ $6.9 \min (n=16)$, referenced to the onset of the VF stimulation.

The centripetal BD shifts of FF neurons evoked by the VF stimulation were always associated with changes in the response magnitude as shown in Figure 4. The responses of neurons at their BDs in the control condition decreased $46 \pm 5.7 \%(n=$ $16)$, whereas the responses at the $\mathrm{BDs}$ in the shifted condition increased $83 \pm$ $13.1 \%(n=16)$. The physiologically meaningful question was whether the increased responses at the shifted BDs were larger than the responses at the control BDs before the electric stimulation. Therefore, in the 16 neurons showing a centripetal $\mathrm{BD}$ shift, the percentage change in the response at the $\mathrm{BD}_{\mathrm{s}}$ was calculated referring to the response at the $\mathrm{BD}_{\mathrm{c}}$ before the VF stimulation. The responses at the new (shifted) BDs were $15 \pm$ $1.2 \%(n=16)$ smaller than the responses at the original (control) $\mathrm{BDs}$ on the average, suggesting that the synapses for the new BDs were not completely mature for synaptic transmission.

The two BD-matched FF neurons did not shift their BDs for electric stimulation of VF neurons and showed facilitation of their responses at their BDs. Figure $4 B$ shows the responses of a FF neuron tuned to a $1.5 \mathrm{~ms}$ delay to paired $\mathrm{FM}_{1}-\mathrm{FM}_{2}$ sounds before, $67 \mathrm{~min}$ after, and $107 \mathrm{~min}$ after the onset of the electric stimulation of the matched VF neurons. Facilitation of the response of the FF neuron was largest at $1.5 \mathrm{~ms}$, so that the delayresponse curve of this FF neuron became sharper after the VF stimulation (Fig. 4 B2, filled circles). The response of the FF neuron at its $\mathrm{BD}$ increased $91 \%$. The response of the neuron reverted to that in the control condition $107 \mathrm{~min}$ after the onset of the stimulation. The other BD-matched FF neuron showed the same changes as those described above for the VF stimulation. However, the amount of the response increase at its $\mathrm{BD}_{\mathrm{c}}$ was not so large, $24 \%$.

\section{Modulation of DF neurons by contralateral DF neurons}

Since electric stimulation of FF neurons evokes centrifugal BD shifts of BD-unmatched FF neurons in the contralateral auditory cortex (Tang et al., 2007), we examined whether electric stimulation of DF neurons evoked the centrifugal or centripetal BD shifts of the BD-unmatched DF neurons in the contralateral auditory cortex. In three mustached bats, we studied a single unit and the evoked potentials at six locations. The single DF neuron (Fig. $5 A$ ) and the six DF evoked potentials (Fig. $5 B$ ) both showed a centrip-
B

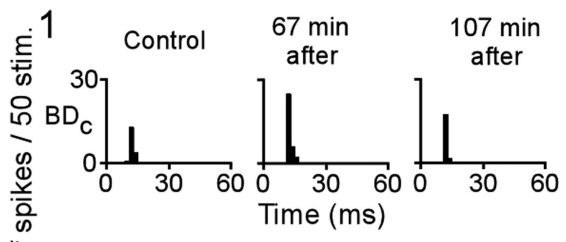

范

은

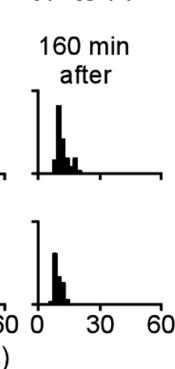

2

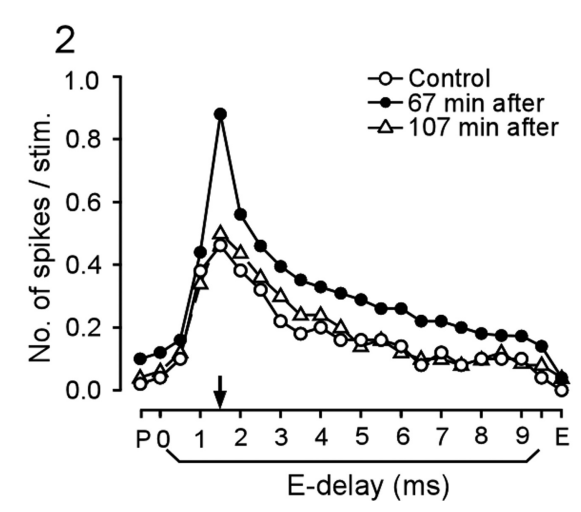

E-delay (ms)

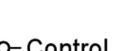
$\rightarrow-90$ min after $-\triangle 160$ min after
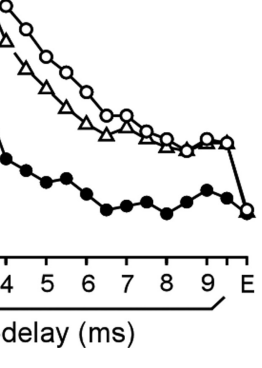

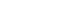

Changes in the responses and delay-response curves of a single BD-unmatched $(\boldsymbol{A})$ and a single BD-matched $(\boldsymbol{B}) \mathrm{FF}$ of $\mathrm{FM}_{1}$ and $\mathrm{FM}_{2}$. $\boldsymbol{A}$, The best delays of the recorded $\mathrm{FF}$ and stimulated $\mathrm{VF}$ neurons were 3.5 and $2.0 \mathrm{~ms}$, respectively. $\boldsymbol{B}$, The BDs eurons both were $1.5 \mathrm{~ms}$. See Figure 2 legend for abbreviations and symbols.

etal BD shift for the contralateral DF stimulation. All the BD shifts obtained from the contralateral DF area are plotted as a function of the BD differences between the recorded and stimulated DF neurons in Figure $3 C$. The slope of the $\mathrm{BD}$ shiftdifference curve in Figure $3 C$ was -0.67 , and the correlation coefficient was 0.97 . Therefore, the data unequivocally indicate that the contralateral DF projection evoked centripetal BD shifts, although the sample number was small. These centripetal BD shifts were the same as those of the DF neurons evoked by electric stimulation of the ipsilateral DF neurons (Tang and Suga, 2008).

\section{Discussion}

BD shifts of three types of FM-FM neurons evoked by electric stimulation

Electric stimulation of FF neurons evokes centrifugal BD shifts of FF neurons in the ipsilateral (Xiao and Suga, 2004) and contralateral (Tang et al., 2007) FF areas, regardless of the differences in FM-FM combination sensitivity between the recorded and stimulated FF neurons. In our current data, combination sensitivity was different in seven pairs of the stimulated FF and recorded VF neurons, but the VF neurons showed the centrifugal BD shift. In two of these seven pairs, the stimulated $\mathrm{FM}_{1}-\mathrm{FM}_{2}$ neurons in the $\mathrm{FF}$ area evoked centrifugal BD shifts of two $\mathrm{FM}_{1}-\mathrm{FM}_{3}$ neurons recorded in the VF area. Therefore, although the sample number was small, our current data support the claim that electric stimulation of FF neurons evoked centrifugal BD shifts regardless of the difference in combination sensitivity between the recorded and stimulated neurons. The anatomical basis for such modulation, however, has not yet been explored, although the mutual projections between the FF, DF, and VF areas were studied by Fitzpatrick et al. (1998). The three components of an echo $\left(\mathrm{FM}_{2}\right.$, $\mathrm{FM}_{3}$, and $\mathrm{FM}_{4}$ ) always return to the bat at the same time from a target after an emission of a biosonar pulse. Therefore, it makes sense that all three types of FM-FM neurons are simultaneously 

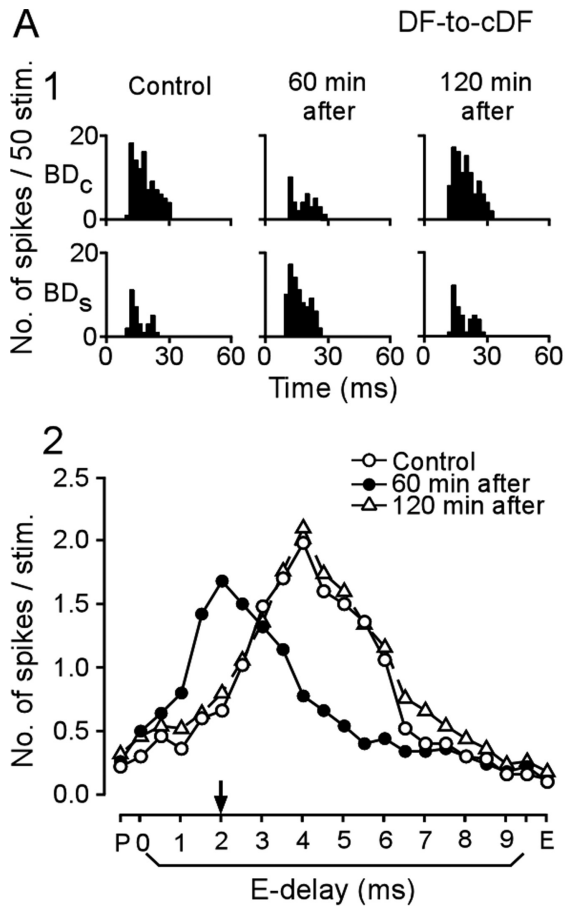

B
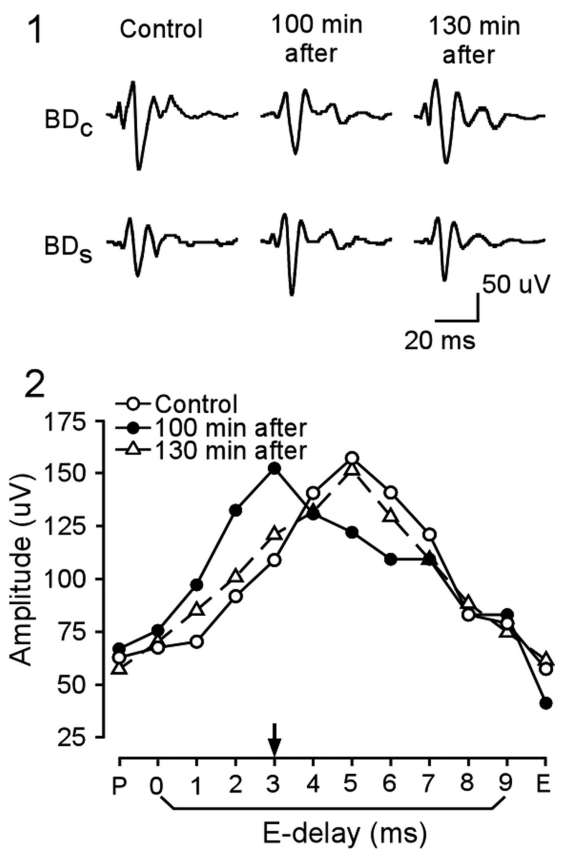

Figure 5. Changes in the responses and delay-response curves of a single DF neuron $(\boldsymbol{A})$ and a DF evoked potential $(\boldsymbol{B})$ evoked by electric stimulation of contralateral BD-unmatched DF neurons. $A$, The DF neuron was tuned to a $\mathrm{FM}_{1}-\mathrm{FM}_{2}$ with a 4.0 ms delay, and the stimulated contralateral DF neurons were tuned to $\mathrm{FM}_{1}-\mathrm{FM}_{2}$ with a $2.0 \mathrm{~ms}$ delay. $\boldsymbol{B}$, The DF evoked potential was tuned to a $F_{1}-F_{2}$ with a 5.0 ms delay, and the stimulated contralateral DF neurons were tuned to a $F_{1}-F_{2}$ with a 3.0 ms delay. See Figure 2 legend for abbreviations and symbols.

modulated in the amount and direction of BD shifts by the stimulated FM-FM neurons.

The VF area represents $0.5-4 \mathrm{~ms}$ BDs. In our current study, the BDs of the stimulated VF neurons were $2.2 \pm 0.27 \mathrm{~ms}(n=$ 18 ) in the mean and SE. Therefore, these VF neurons were mostly located at the center of the VF area. The electric stimulus presumably spread $\sim 10 \%$ within the VF area. Electric stimulation of VF neurons also evoked the BD shifts of FF neurons regardless of the differences in FM-FM combination sensitivity between the recorded FF and stimulated VF neurons. However, it might influence different types of FM-FM combination-sensitive neurons in the VF area and might evoke the BD shifts of those in the FF area.

Modulation of BD-matched and -unmatched neurons by focal electric stimulation of the delay-tuned auditory cortex

Electric stimulation of FF neurons evokes facilitation of subcortical BD-matched delay-tuned (FM-FM) neurons at their BDs and the BD shifts of subcortical BD-unmatched delay-tuned neurons away from the $\mathrm{BD}$ of the stimulated neurons. This basic function has been called "egocentric selection" (Yan and Suga, 1996). BD-unmatched neurons are easily sampled, but BDmatched neurons are hardly sampled without special effort to do so. In our current studies, there were only two BD-matched FF neurons, so that one may doubt whether the facilitation of the $\mathrm{BD}$-matched neurons was a general phenomenon. In the past, Suga and his collaborators have recorded the BF-matched neurons in the inferior colliculus (Zhang et al., 1997; Zhang and Suga, 2000), medial geniculate body (Zhang et al., 1997; Zhang and Suga, 2000), and auditory cortex (Xiao and Suga, 2005), best duration-matched neurons in the inferior colliculus (Ma and Suga, 2001), and BD-matched neurons in the inferior colliculus (Yan and Suga, 1996) and the auditory cortex (Xiao and Suga,

2004; Tang et al., 2007). All these tuningmatched neurons show facilitation of their responses and sharpening and no shift of their tuning for electric stimulation of cortical auditory neurons. Therefore, we can claim that our data obtained from the two BD-matched neurons are not exceptional, and that the BD-matched neurons do not show a BD shift, because all three regression lines in Figure 3 crossed the origin of the coordinates. As a matter of fact, the two BD-matched FF neurons studied in our current research showed no BD shifts.

In addition to facilitation of the $\mathrm{BD}$ matched neurons, centrifugal or centripetal BD shifts of BD-unmatched neurons are evoked by electric stimulation of the cortical delay-tuned neurons, as reviewed below. Electric stimulation of FF neurons evokes the centrifugal BD shifts of nearby FF neurons through the lateral projection (Xiao and Suga, 2004), contralateral FF neurons through the contralateral projection (Tang et al., 2007), ipsilateral DF (Tang and Suga, 2008) and VF (our current study) neurons through feedforward projections, and ipsilateral subcortical delay-tuned neurons through the corticofugal projections (Yan and Suga, 1996). These centrifugal BD shifts elicited by the FF stimulation are small, only up to $2.0 \mathrm{~ms}$

(Fig. 6A).

Electric stimulation of DF neurons evokes the centripetal BD shifts of nearby DF neurons (Tang and Suga, 2008), contralateral DF neurons (our current paper), and FF neurons (Tang and Suga, 2008) through the lateral, contralateral, and feedback projections, respectively. The BD shifts elicited by the DF neurons were large, up to $5.0 \mathrm{~ms}$ (Fig. $6 B$ ).

Electric stimulation of VF neurons evokes the centripetal BD shifts of FF neurons through the feedback projection. The BD shifts evoked by VF neurons were also large, up to $4.5 \mathrm{~ms}$ (Fig. $6 C)$. The BD shifts summarized above are also explained by the block diagram in Figure $6 D$, in which the arrows indicate the projections evoking the centripetal BD shifts, whereas the lines with a bar at the end indicate those evoking the centrifugal $\mathrm{BD}$ shifts. The interactions between ipsilateral and contralateral VF neurons and those between DF and VF neurons have not yet been studied, because recording action potentials of VF neurons was not easy due to the small size of the VF area and because the mustached bat became unavailable to us. The electric stimulation of the FF area and DF area, respectively, evokes the centrifugal and centripetal BD shifts regardless of their projections (Fig. 6D). Therefore, we may speculate that the ipsilateral and contralateral VF projections and the DF-to-FF projection all evoke centripetal BD shifts.

One may consider that our data are not conclusive because of the small sample size of the neurons studied. However, the correlation coefficients for the regression lines in Figure 3 were 0.94 for $\mathrm{A}, 0.98$ for $\mathrm{B}$, and 0.97 for $\mathrm{C}$. That is, the variation of the data points plotted in Figure 3 was very small. Therefore, our claims are valid that the FF-to-VF projection elicited centrifugal $\mathrm{BD}$ shifts, whereas the VF-to-FF and DF-to-cDF projections elicited 
centripetal BD shifts. If the variation were large, the sample size should be large to make such a claim.

\section{Functional significance of the centripetal and centrifugal BD shifts}

The centrifugal BD shifts increase the contrast or selectivity in the neural representation of a specific target distance, whereas the centripetal BD shifts increase the population of neurons tuned to that specific target distance. The centripetal BD shifts elicited by the feedback projections from the DF and VF areas to the FF area were 2.6 times larger than the centrifugal BD shifts elicited by the feedforward projections from the FF area to the DF and VF areas (Fig. 6). The large BD shift of the FF neurons for the electric stimulation of the DF and VF neurons indicate that the excitation of the DF and VF neurons tuned to a given delay can dramatically increase the number of FF neurons tuned to that delay through the feedback projections. Therefore, we may hypothesize that the DFto-FF and VF-to-FF feedback projections play a role in focusing the neural processing of target information at a specific distance represented by the FF area.

The FF area represents the target distances up to $310 \mathrm{~cm}$ (Suga and O'Neill, 1979). The DF and VF areas respectively represent the target distances shorter than $140 \mathrm{~cm}$ (Suga and O'Neill, 1979) and 70 cm (Suga and Horikawa, 1986). During a target-directed flight, an echo delay systematically shortens. The neural representations of the target distances in the FF area are presumably enhanced by the DF area during the approach phase of the flight and by both the DF and VF areas during the terminal phase of the flight for finer analysis of a target at shorter distances. Unlike echo delays during target-directed flight, echo delays are short during exploration of nearby objects during roosting in a cave. In such a situation, the FF area may also play a role in responding to a specific echo delay and the DF and VF areas may play a role in enhancing the response to or focusing at that specific echo delay.

\section{The origin of the modulation (i.e., egocentric selection) evoked by cortical electric stimulation}

There are three possibilities of the origin of the egocentric selection elicited by the electric stimulation: (1) the egocentric selection occurs in the electrically stimulated cortical area and is simply transferred to the target areas, (2) the egocentric selection occurs in the target areas, and (3) both of the above.

Focal electric stimulation of the primary auditory cortex (AI) and auditory fear conditioning both evoke the BF shifts in AI and the central nucleus of the inferior colliculus (for review, see Suga and $\mathrm{Ma}, 2003)$. The direction of the collicular BF shift elicited by the focal electric stimulation of AI can be changed by a cortical application of an agonist or antagonist of $\mathrm{GABA}_{\mathrm{A}}$ receptors $(\mathrm{Ma}$ and Suga, 2004; Xiao and Suga, 2005). Inactivation of AI abol-
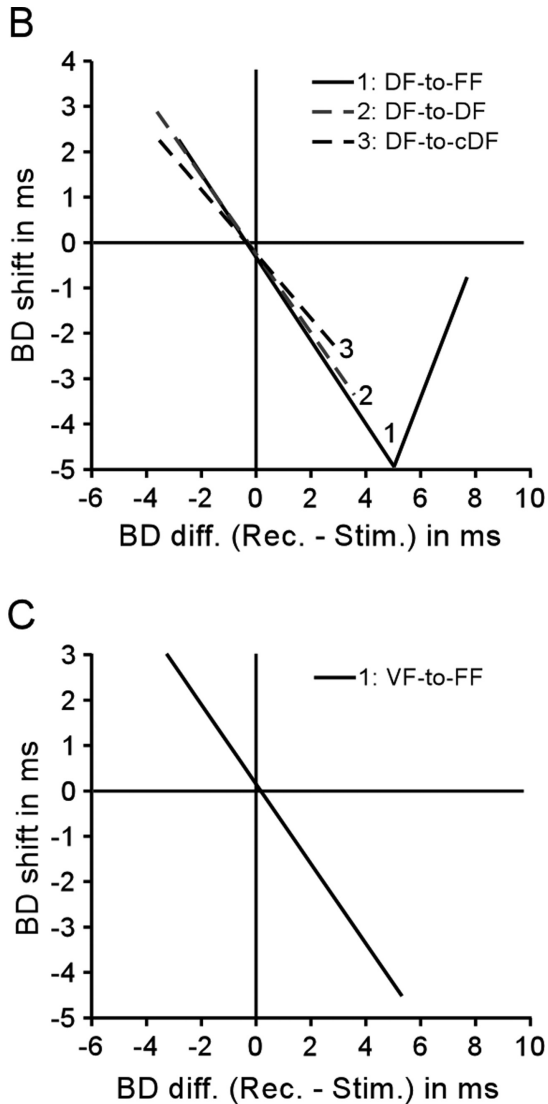

Figure 6. The BD shift-difference curves for the BD shifts evoked by electric stimulation of either the $F F(\boldsymbol{A}), \mathrm{DF}(\boldsymbol{B})$, or VF $(\boldsymbol{C})$ eurons and the block diagram showing feedforward, feedback, lateral, and contralateral modulations $(\boldsymbol{D}) . \boldsymbol{A}$, "1-5" respectively (o evoked. This summary figure is based on the data obtained by Yan and Suga (1996), Xiao and Suga (2004), Tang et al. (2007), Tang and Suga (2008), and our current studies.

ishes the development of the conditioning-dependent collicular BF shift without affecting the responses to tone bursts and frequency tuning of the collicular neurons (Gao and Suga, 1998, 2000). Electric stimulation of the central nucleus of the inferior colliculus (Zhang and Suga, 2005) and the ventral division of the medial geniculate body ( Wu and Yan, 2007) evokes the collicular $\mathrm{BF}$ shift, which is abolished by inactivation of AI. Electric stimulation of FF neurons evokes the centrifugal $\mathrm{BD}$ shifts of the FF neurons located near the stimulated ones. As the BF shifts, these BD shifts change from centrifugal to centripetal when cortical inhibition is removed by an application of an antagonist of $\mathrm{GABA}_{\mathrm{A}}$ receptors to the stimulated site (Xiao and Suga, 2004). Therefore, it is most likely that the BD shifts observed in our series of experiments mostly, if not totally, originate from the changes in the electrically stimulated cortical area and are transmitted to the other cortical auditory areas and subcortical auditory nuclei.

\section{Comparative aspects of tuning shifts}

The corticocortical interaction within a specific cortical area has been studied in AIs of different mammalian species [rats, Talwar and Gerstein (2001); big brown bats, Chowdhury and Suga (2000) and Ma and Suga (2001); Mongolian gerbils and mus- 
tached bats, Sakai and Suga (2001)], the primary visual cortex [cats, Godde et al. (2002) and Calford et al. (2003)], and the primary somatosensory cortex [rats and monkeys, Recanzone et al. (1992); human, Münchau et al. (2002)]. In all these cortices and species of animals, focal cortical electric stimulation evoked the centripetal shifts of the receptive fields (or tuning curves) of neurons surrounding the stimulated neurons.

In the mustached bat, electric stimulation of the posterior division of $\mathrm{AI}$ evokes centripetal $\mathrm{BF}$ shifts of nearby neurons (Sakai and Suga, 2001). However, electric stimulation of the DSCF (Zhang and Suga, 2000; Xiao and Suga, 2005) and FF (Xiao and Suga, 2004) neurons respectively evoked centrifugal BF or BD shifts of nearby DSCF and FF neurons. The DSCF and FF areas are highly specialized for processing specific biosonar information (for review, see Suga, 1990). Therefore, corticocortical interactions within the DSCF or FF areas are unique. In animal species other than the mustached bat, highly specialized cortical auditory areas have not yet been identified, and the corticocortical interactions have only superficially been studied. With the data thus far available, one may conclude that the centripetal tuning shifts are much more common than the centrifugal tuning shifts.

The interaction between different cortical areas has thus far been studied only in the cat visual cortex (Galuske et al., 2002). The deactivation of the visuoparietal cortex decreases orientation and direction sensitivities of neurons in area 18 and abolishes the global layout of the direction map in it. The higher cortical visual area significantly contributes to the creation of the basic response properties of the lower cortical visual area. On the other hand, our current and previous data (Tang and Suga, 2008) indicate that the activation of the VF and DF areas facilitates the matched FF neurons and shifts the delay tuning of the unmatched FF neurons. The basic response properties (delay tuning) of the FF neurons are presumably established without the DF-to-FF and VF-to-FF feedback projections, because thalamic and collicular FM-FM neurons are tuned to specific echo delays.

\section{References}

Calford MB, Wright LL, Metha AB, Taglianetti V (2003) Topographic plasticity in primary visual cortex is mediated by local corticocortical connections. J Neurosci 23:6434-6442.

Chowdhury SA, Suga N (2000) Reorganization of the frequency map of the primary auditory cortex evoked by focal cortical electrical stimulation in the big brown bat. J Neurophysiol 83:1856-1863.

Edamatsu H, Kawasaki M, Suga N (1989) Distribution of combinationsensitive neurons in the ventral fringe area of the auditory cortex of the mustached bat. J Neurophysiol 61:202-207.

Fitzpatrick DC, Olsen JF, Suga N (1998) Connections among functional areas in the mustached bat auditory cortex. J Comp Neurol 391:366-396.

Galuske RA, Schmidt KE, Goebel R, Lomber SG, Payne BR (2002) The role of feedback in shaping neural representations in cat visual cortex. Proc Natl Acad Sci U S A 99:17083-17088.

Gao E, Suga N (1998) Experience-dependent corticofugal adjustment of midbrain frequency map in bat auditory system. Proc Natl Acad Sci U S A 95:12663-12670.

Gao E, Suga N (2000) Experience-dependent plasticity in the auditory cortex and the inferior colliculus of bats: role of the corticofugal system. Proc Natl Acad Sci U S A 97:8081-8086.

Godde B, Leonhardt R, Cords SM, Dinse HR (2002) Plasticity of orientation preference maps in the visual cortex of adult cats. Proc Natl Acad Sci U S A 99:6352-6357.

Ma X, Suga N (2001) Plasticity of bat's central auditory system evoked by focal electric stimulation of auditory and/or somatosensory cortices. J Neurophysiol 85:1078-1087.

Ma X, Suga N (2004) Lateral inhibition for center-surround reorganization of the frequency map of bat auditory cortex. J Neurophysiol 92:3192-3199.

Misawa H, Suga N (2001) Multiple combination-sensitive neurons in the auditory cortex of the mustached bat. Hear Res 151:15-29.

Münchau A, Bloem BR, Irlbacher K, Trimble MR, Rothwell JC (2002) Functional connectivity of human premotor and motor cortex explored with repetitive transcranial magnetic stimulation. J Neurosci 22:554-561.

O'Neill WE, Suga N (1982) Encoding of target-range information and its representation in the auditory cortex of the mustached bat. J Neurosci 2:17-31.

Recanzone GH, Merzenich MM, Dinse HR (1992) Expansion of the cortical representation of a specific skin field in primary somatosensory cortex by intracortical microstimulation. Cereb Cortex 2:181-196.

Rouiller E (1997) Functional organization of the auditory pathway. In: The central auditory system (Ehret G, Romand R, ed), pp 43-45. New York: Oxford UP.

Sakai M, Suga N (2001) Plasticity of the cochleotopic (frequency) map in specialized and nonspecialized auditory cortices. Proc Natl Acad Sci U S A 98:3507-3512.

Suga N (1990) Cortical computational maps for auditory imaging. Neural Netw 3:3-21.

Suga N, Horikawa J (1986) Multiple time axes for representation of echo delays in the auditory cortex of the mustached bat. J Neurophysiol $55: 776-805$.

Suga N, MaX (2003) Multiparametric corticofugal modulation and plasticity in the auditory system. Nat Rev Neurosci 4:783-794.

Suga N, O’Neill WE (1979) Neural axis representing target range in the auditory cortex of the mustached bat. Science 206:351-353.

Suga N, Gao E, Zhang Y, Ma X, Olsen JF (2000) The corticofugal system for hearing: recent progress. Proc Natl Acad Sci U S A 97:11807-11814.

Suga N, Xiao Z, Ma X, Ji W (2002) Plasticity and corticofugal modulation for hearing in adult animals. Neuron 36:9-18.

Talwar SK, Gerstein GL (2001) Reorganization in awake rat auditory cortex by local microstimulation and its effect on frequency-discrimination behavior. J Neurophysiol 86:1555-1572.

Tang J, Suga N (2008) Modulation of auditory processing by corticocortical feed-forward and feedback projections. Proc Natl Acad Sci U S A 105:7600-7605.

Tang J, Xiao Z, Suga N (2007) Bilateral cortical interaction: modulation of delay-tuned neurons in the contralateral auditory cortex. J Neurosci 27:8405-8413.

Wu Y, Yan J (2007) Modulation of the receptive fields of midbrain neurons elicited by thalamic electrical stimulation through corticofugal feedback. J Neurosci 27:10651-10658.

Xiao Z, Suga N (2002) Reorganization of the cochleotopic map in the bat's auditory system by inhibition. Proc Natl Acad Sci U S A 99:15743-15748.

Xiao Z, Suga N (2004) Reorganization of the auditory cortex specialized for echo-delay processing in the mustached bat. Proc Natl Acad Sci U S A 101:1769-1774.

Xiao Z, Suga N (2005) Asymmetry in corticofugal modulation of frequencytuning in mustached bat auditory system. Proc Natl Acad Sci U S A 102:19162-19167.

Yan J, Suga N (1996) Corticofugal modulation of time-domain processing of biosonar information in bats. Science 273:1100-1103.

Yan W, Suga N (1998) Corticofugal modulation of midbrain frequency map in bat auditory system. Nat Neurosci 1:54-58.

Zhang Y, Suga N (2000) Modulation of responses and frequency tuning of thalamic and collicular neurons by cortical activation in mustached bats. J Neurophysiol 84:325-333.

Zhang Y, Suga N (2005) Corticofugal feedback for collicular plasticity evoked by electric stimulation of the inferior colliculus. J Neurophysiol 94:2676-2682.

Zhang Y, Suga N, Yan J (1997) Corticofugal modulation of frequency processing in bat auditory system. Nature 387:900-903. 